\title{
Conmigo o contra mí: análisis de la concordancia y las estrategias temáticas del Centro Democrático en Twitter
}

\section{Carlos Felipe Cifuentes ${ }^{1}$ Juan Federico Pino ${ }^{2}$}

Recibido: 2017-08-12

Enviado a pares: 2017-10-15
Aprobado por pares: 2017-10-15

Aceptado: 2017-20-11

DOI: $10.5294 /$ pacla.2018.21.3.10

Para citar este artículo / to reference this article / para citar este artigo Cifuentes, C. F. y Pino, J. F. (2018). Conmigo o contra mí: análisis de la concordancia y estrategias temáticas del Centro Democrático en Twitter. Palabra Clave, 21(3), 885-916. DOI: 10.5294/pacla.2018.21.3.10

\section{Resumen}

Twitter es una herramienta cada vez más utilizada por los políticos para comunicarse con sus seguidores y definir sus principales posturas ideológicas. Este artículo identifica si los congresistas del Centro Democrático tienen una agenda homogénea, identificándose con su partido político, o heterogénea buscando posicionar su imagen sobre la de sus copartidarios, según los supuestos de la teoría de la identidad y del personalismo político. Para ello, se recolectó, a través del media mining, 114777 tuits de las cuentas de los congresistas del Centro Democrático, que se analizaron cuantitativamente para testear las hipótesis de investigación. El estudio permitió observar que los congresistas del Centro Democrático, como se esperaba desde la teoría de la identidad social, tienen una agenda temática homogénea caracterizada por utilizar una estrategia de exogrupo que identifica al presidente Juan Manuel Santos y las Fuerzas Armadas Revolucionarias de

1 orcid.org/0000-0002-1975-1969. Universidad de los Andes, Colombia. cf.cifuentes10@uniandes.edu.co

2 orcid.org/0000-0003-0799-3001. Universidad de los Andes, Colombia.jf.pino31@uniandes.edu.co 
Colombia como principales antagonistas, y una estrategia de endogrupo que identifica al expresidente Álvaro Uribe y al partido como principales figuras de identificación colectiva.

\section{Palabras clave}

Twitter; comunicación política; agenda temática; endogrupo; exogrupo (Fuente: Tesauro de la Unesco). 


\section{With Me or Against Me: Analysis of the Concordance and Thematic Strategies of the Centro Democrático Political Party on Twitter}

\section{Abstract}

Twitter is a tool increasingly used by politicians to communicate with their followers and to define their main ideological positions. This paper identifies whether the congressmen of the Centro Democrático have a homogeneous agenda in which they identify with their political party, or a heterogeneous agenda, in which they seek to position their image over that of the other members of their party, based on the theory of identity and political personalism. To that end, we used media mining to collect 114,777 tweets from the accounts of Centro Democrático congressmen, which were analyzed quantitatively to test the research hypothesis. The study showed that, as expected from the theory of social identity, the Centro Democrático congressmen have a homogeneous thematic agenda characterized by using an outgroup strategy that identifies President Juan Manuel Santos and the Revolutionary Armed Forces of Colombia as the main antagonists and an endo-group strategy that identifies former President Alvaro Uribe and that party as the main figures of collective identification.

\section{Keywords}

Twitter; political communication; thematic agenda; endo-group; outgroup (Source: Unesco Thesaurus). 


\section{Comigo ou contra mim: análise da concordância e das estratégias temáticas do Centro Democrático no Twitter}

\section{Resumo}

O Twitter é uma ferramenta cada vez mais utilizada pelo políticos para se comunicar com seus seguidores e definir suas principais posturas ideológicas. Este artigo identifica se os candidatos do Centro Democrático têm uma agenda homogênea, na qual se identificam com seu partido político, ou heterogênea, na qual buscam posicionar sua imagem sobre a dos outros membros do partido, baseado na teoria da identidade e do personalismo público. Para isso, 114.777 tuítes das contas dos candidatos do Centro Democrático foram coletados através de media mining, os quais foram analisados quantitativamente para testar as hipóteses da pesquisa. O estudo permitiu observar que os congressistas do Centro Democrático, como era esperado de acordo com a teoria da identidade social, têm uma agenda temática homogênea caracterizada por utilizar uma estratégia de exogrupo, a qual identifica ao presidente Juan Manuel Santos e às Forças Armadas Revolucionárias da Colômbia como principais antagonistas, e uma estratégia de endogrupo, a qual identifica o ex-presidente Álvaro Uribe e o partido como principais figuras de identificação coletiva.

\section{Palavras-chave}

Agenda temática; comunicação política; endogrupo; exogrupo; Twitter (Fonte: Tesauro da Unesco). 


\section{Introducción}

La frecuencia en el uso de las redes sociales por parte de los partidos ${ }^{3}$ y políticos ha cambiado significativamente durante la última década en todo el mundo. Las redes sociales, en general, han desempeñado un creciente papel en la forma en que los partidos y políticos se comunican y transmiten sus principales posturas personales o partidistas a sus seguidores y a la ciudadanía (Resimić, 2016; Cogburn y Espinoza-Vasquez, 2011; Hanson, Haridakis, Cunningham, Sharma y Ponder, 2010; Túñez y Sixto, 2011). Este cambio en las formas de comunicación, que es uno de los ejes centrales de la sociedad y la actividad política (Becerra Garduño, 2015; Luhmann, 1990), ha significado que partidos y políticos se tengan que adaptar a las nuevas herramientas tecnológicas. Estas, a diferencia de la radio o la televisión, permiten a los políticos comunicarse mediáticamente con los ciudadanos sin la intermediación del partido o la necesidad de grandes medios de comunicación (Thompson, 1998). A su vez, las redes sociales han debilitado la capacidad de los partidos de proyectar una única agenda temática que los identifique colectivamente (Ross y Bürger, 2014).

En el presente estudio, se investiga el uso de Twitter por parte de los políticos del Centro Democrático, enfocándose en la forma en que estos utilizaron sus tuits para construir una agenda temática común o para posicionarse individualmente. Es importante comprender este comportamiento en Twitter, porque los mensajes partidistas, en esta red social, sirven como un medio primario en la forma en que los ciudadanos socializan políticamente y construyen sus opiniones e identidades sobre asuntos políticos ( Knowles, Lowery, Shulman y Schaumberg, 2013). En este sentido, una agenda más homogénea permitiría a los ciudadanos identificar más fácilmente los partidos políticos y sus propuestas ideológicas, mientras que una agenda política heterogénea haría más difícil la identificación de los

3 El partido político es entendido como "cualquier grupo político que se presenta a competir en elecciones y que puede colocar mediante ellas a sus candidatos en cargos públicos” (Sartori, 1980, p. 20) bajo una etiqueta que lo identifique respecto de los otros grupos. Los partidos se pueden desagregar en tres componentes: 1) el partido en el Gobierno, 2) los trabajadores políticos y 3) el partido en el electorado (Issacharoff, 2016; Persily y Cain, 2000). En este artículo, solo se estudia el partido en el Gobierno nacional; como actualmente el Centro Democrático está en oposición, solo se analiza a los miembros del partido que fueron elegidos popularmente a nivel nacional, en este caso los congresistas. 
partidos por sus temas e incidiría en que el voto de los ciudadanos fuera más personalista (Carey y Shugart, 1995). Por esto mismo, un partido con una agenda temática más homogénea puede ser un comunicador efectivo respecto de sus propuestas e incentivar un voto más programático (Mainwaring y Bejarano, 2008; Wattenberg, 1982). Mientras que un partido con una agenda más heterogénea estaría reflejando una comunicación en la que los políticos buscan un posicionamiento individual, lo cual puede incentivar y reflejar prácticas más particularistas e incluso clientelistas para movilizar el voto ciudadano (Roth, Peters y Seruga 2014; Hoskin y García, 2006; Lancaster, 1986).

Este artículo explora los tuits $(\mathrm{n}=113.747)$ que producen los miembros del Centro Democrático para observar si los políticos muestran una agenda temática común en los mensajes que transmiten en Twitter o esta es individual. Se consideran dos marcos que ofrecen una perspectiva sobre el comportamiento colectivo o individual en Twitter: la teoría de la identidad social (Tajfel y Turner, 1979) y la del personalismo partidista (Carey y Shugart, 1995). La teoría de la identidad social (Tajfel y Turner, 1979) expone que la identidad de un individuo se basa en gran parte en la pertenencia a un grupo; las percepciones de esa identidad pueden crearse o incrementarse atacando a los grupos externos. Mientras que la teoría personalista sostiene que, en contextos institucionales que incentivan el voto personalista, los políticos van a posicionar su imagen para distinguirse de sus copartidarios, por lo cual el partido tendría una agenda temática en Twitter más heterogénea (Novaes, 2015; Edwards y Thames, 2007).

\section{Entre la identidad social y el personalismo}

Las teorías de la identidad y el personalismo, a pesar de no ser construidas en torno a la interacción mediática en redes sociales, ofrecen distintas explicaciones teóricas de la forma en que los políticos realizan acciones para proyectarse ante sus seguidores. Esta teoría sostiene que la afiliación grupal sirve como una forma en que la gente, y en este caso los partidos y políticos, busca proyectarse. Los políticos pueden mejorar la imagen de su colectividad a través de la identificación social con un endogrupo y la comparación social con un exogrupo. Aunque esta teoría no se ha desarrollado especí- 
ficamente para entender situaciones políticas, puede aplicarse también a este tipo de análisis (Kenski, Filer y Conway, 2017). Por su parte, la teoría del personalismo sostiene que en contextos donde se incentiva el voto personalista, como es el colombiano (García y Wills, 2011; Hoskin y García, 2006), las acciones proselitistas no buscarían hacer referencia a los temas de su partido, sino a temáticas individuales para posicionar su propia imagen.

Por su parte, la teoría de la identidad sugiere que los políticos buscan producir sus mensajes en torno a un proceso de identificación social, por ello los mensajes contribuyen a definir antagonistas y a identificar al propio grupo de pertenencia, "lo que en la práctica se materializa en la selección de vocablos con los que se representa de manera positiva al propio grupo y negativamente al contrario" (Adrián, 2013, p. 10). Estas estrategias permiten asociar rasgos negativos respecto del exogrupo (ellos) e identificar y dar rasgos positivos al endogrupo (nosotros). Este uso del lenguaje posibilita la formación de estereotipos a través, en este caso, de asociaciones de palabras negativas, respecto de los actores rivales (Becerra, 2015).

Las investigaciones actuales sobre el comportamiento en Twitter de los partidos políticos en los Estados Unidos y América Latina indican un aumento del "partidismo negativo", en el cual los miembros de cada partido tienden a producir mensajes negativos de los opositores (Abramowitz y Webster, 2015; Iyengar, Sood y Lelkes, 2012; Bafumi y Sapiro, 2009; Iyengar, 2011), y así muestran que, al menos en este aspecto, los políticos de un mismo partido tienen una agenda temática en común.

Aharony (2012) sostiene que el sentido de pertenencia de un grupo puede ser fortalecido a través de la hostilidad hacia los grupos que estos identifican como externos. Esto aumenta la posibilidad de que los políticos de un partido tengan un comportamiento similar en Twitter y que sus mensajes tiendan a reflejar una postura colectiva más que individual en esta red social (Alhabash y McAlister, 2014). Una forma en que los políticos pueden mostrar hostilidad fuera del grupo es atacando a la parte contraria, por ejemplo, al presidente en funciones o miembros reconocidos de otros partidos políticos distintos del suyo. En este orden de ideas, según esta teoría, 
se espera que los políticos de un partido hagan referencias al partido contrario y le adjudiquen atributos negativos.

Por otra parte, la teoría del personalismo plantea que en países donde las instituciones electorales hacen que importe más la votación de los políticos que la de su partido para ser elegidos, los políticos buscan posicionar en campaña y en su desempeño en política su imagen particular sobre la de su partido, al tener que competir contra sus copartidarios para ser elegidos más que contra otros partidos (Carey y Shugart, 1995). Los políticos, en este sentido, buscarán apartarse de la agenda de sus partidos para ser identificados individualmente por sus electores. Más aún, en sistemas de partidos como los latinoamericanos que se han caracterizado por tener bajos niveles de institucionalización, donde la etiqueta partidista ha perdido valor (Dosek y Flavia, 2014; Mainwaring y Bejarano, 2008) y que tienen instituciones que incentivan un comportamiento personalista de los políticos sobre los partidos, lo cual debilita la disciplina y coherencia partidista (Botero, 2011; Mainwaring y Scully, 1995). Distintas investigaciones han señalado el comportamiento personalista que varios políticos presentan en Twitter y la falta de coordinación temática de los partidos (Kenski, Filer y Conway, 2017; Adi, Erickson y Lilleker, 2014). Desde esta teoría, se puede esperar, por tanto, que los tuits de los políticos tenderán a reflejar una agenda temática propia más que colectiva, lo cual tendrá como resultado una agenda temática más diversa y menos coordinada entre los miembros de un partido político.

Estas dos teorías sugieren que los intereses de los políticos son importantes en la estructuración de sus mensajes y en la forma que quieren ser percibidos por los ciudadanos. En América Latina, Europa y los Estados Unidos, se ha encontrado variación en la forma en que se comportan los políticos en Twitter, mostrando que, en algunos casos, prima una agenda temática compartida y mensajes en torno a la construcción de identidad (Conway, Kenski y Wang, 2013; Kruikemeier, 2014; Peterson, 2012; Škilters, Kreile, Bojārs, Brikše, Pencis y Uzule, 2012; Straus, Glassman, Shogan y Smelcer, 2013), mientras que en otros se privilegian los mensajes personalistas sobre los de la colectividad (Kenski, Filer y Conway, 2017; Grussel y Nord, 
2012; Small, 2010). En este sentido, desde la teoría de la identidad social, se espera que los mensajes de los miembros de un partido compartan una agenda temática que esté dirigida a identificar su endogrupo con palabras positivas y al exogrupo con palabras negativas. Mientras que desde la teoría del personalismo se espera que los políticos produzcan sus mensajes para aumentar su autoimagen, por lo cual el partido tendría una agenda temática más heterogénea. Según estas expectativas teóricas, este artículo se plantea dos preguntas investigación: ¿se puede identificar en el corpus ${ }^{4}$ de los tuits de las cuentas del Centro Democrático una agenda temática homogénea o heterogénea? y ¿cómo es este proceso de construcción de una agenda homogénea o heterogénea en este partido?

\section{Ellos o nosotros: el caso del Centro Democrático}

Durante las últimas dos décadas, los políticos en Colombia se han caracterizado por tener altos niveles de autonomía frente a sus partidos políticos (Gutiérrez, 2007; Pizarro, 2008) y estos últimos por presentar elevados niveles de fragmentación a nivel nacional y subnacional (Pino, 2017; Milanese, Abadía y Manfredi, 2016). Aunque existen investigaciones sobre el comportamiento colectivo o individual de los miembros de los partidos políticos en el Congreso de la República (Santamaría, 2013; Duque, 2011), este tema ha sido escasamente estudiado en redes sociales y se han enfocado más en la interacción entre votantes y políticos más que en las dinámicas comunicativas del partido (Ramírez-Castro, 2014). Al respecto, Correa y Camargo (2017) hacen una aproximación al comportamiento temático de agrupaciones de partidos y no encuentran diferencias ideológicas ni temáticas entre los miembros de distintos partidos. No obstante, su unidad de análisis son agrupaciones de ellos, no partidos como tal.

El Centro Democrático fue fundado el 15 de julio de 2014. Este partido se ha caracterizado por las relaciones de jerarquía en torno a la figura del expresidente Álvaro Uribe, quien ha ejercido una fuerte oposición

4 El estudio de los corpus es pertinente, ya que, según Sinclair (1991), un corpus es una colección de textos que permite caracterizar un estado o postura respecto de una forma de utilizar el lenguaje de determinado grupo, por lo cual parte de una colección de palabras o expresiones para servir como muestra de la lengua y del empleo que se le da a esta (Charaudeau y Mainguenau, 2002). 
al proceso de paz adelantado por el presidente Juan Manuel Santos con las Fuerzas Armadas Revolucionarias de Colombia (FARC) ${ }^{5}$ (Gunther y Diamond, 2003; Losada y Liendo, 2016; Caicedo, 2016; Velasco, 2017) y ha realizado un fuerte discurso contra la inseguridad y criminalidad en Colombia (Norza, Peñalosa y Rodríguez, 2017; Norza y Espino, 2011). Por otra parte, el sistema electoral colombiano incentiva los comportamientos individuales que propenden al posicionamiento particular de los políticos sobre sus partidos (Shugart y Pachón, 2010). Estas características del Centro Democrático y del sistema electoral colombiano hacen que este partido se constituya en un caso de estudio interesante para analizar las dinámicas en Twitter en torno, específicamente, a la construcción de agendas temáticas homogéneas o heterogéneas, a través del estudio del corpus de los tuits de los congresistas del Centro Democrático.

\section{Hipótesis de investigación}

Estudiar los procesos de construcción de agendas temáticas homogéneas o heterogéneas en Twitter desde la perspectiva de la identidad social (Tajfel y Turner, 1979) y el personalismo político (Carey y Shugart, 1995) permite plantear hipótesis frente a las preguntas de investigación. Según los hallazgos de Correa y Camargo (2017), los políticos tienen una agenda que no se diferencia por agrupaciones de partidos políticos en sus mensajes en Twitter, lo cual permitiría suponer que tienen una agenda individual en esta red social. No obstante, las investigaciones cualitativas han mostrado cómo, desde distintas redes sociales, los congresistas del Centro Democrático han utilizado el ataque como estrategia, principalmente hacia el Gobierno de Juan Manuel Santos y a las FARC (Velasco, 2017; Caicedo, 2016). Esto permitiría suponer la existencia de una agenda temáticamente homogénea.

Según estos planteamientos, se formulan las siguientes hipótesis:

5 Las negociaciones de paz entre el Gobierno fueron las conversaciones que el Gobierno del presidente Juan Manuel Santos (2010-2018) adelantó con las FARC, la guerrilla más antigua de este país. Estos diálogos se realizaron en Oslo, Noruega y La Habana, y finalizaron con la firma del acuerdo de paz en el Teatro Colón en Bogotá, el 24 de noviembre de 2016. 
- $\quad \mathrm{H} 1$ : El corpus de los tuits de las cuentas de los congresistas del Centro Democrático se caracteriza por mostrar una agenda temática heterogénea en torno a las posturas individuales de los congresistas.

- H2: El corpus de los tuits de las cuentas de los congresistas del Centro Democrático se caracteriza por mostrar una agenda temática homogénea en torno a la identificación de un exogrupo y endrogupo.

\section{Datos y metodología}

La información utilizada en este artículo se obtuvo directamente de las cuentas de Twitter de los congresistas del Centro Democrático. Los datos están disponibles en línea y su acceso es abierto al público. En primera instancia, se identificó si los 20 senadores, 19 representantes a la Cámara y el partido tenían cuenta vigente en Twitter. Una vez establecida la autenticidad de las 40 cuentas, se recolectaron aleatoriamente 3000 tuits de cada una, para $114777^{6}$ tuits por medio del programa Twilet. ${ }^{7}$ Los tuits fueron recolectados entre el 4 de marzo de 2012 y el 30 de junio de 2017.

Los tuits recolectados fueron sometidos a un proceso de edición donde se retiraron las arrobas, los numerales y las HTTP para facilitar el análisis textual. A continuación, se utilizó el freeware AntConc ${ }^{8}$ para hacer un análisis del corpus mediante la identificación, cuantificación y sistematización de las palabras más utilizadas en las distintas cuentas. Una vez recolectada y sistematizada la información, el proceso de análisis se organizó en dos fases definidas por las preguntas de investigación.

En la primera fase, se examinó el corpus de cuentas del Centro Democrático para identificar el nivel de concordancia temática en el discurso

6 La cifra estimada de tuits, si todas las cuentas tuvieran la misma producción, sería de 120 000; no obstante, algunas cuentas tienen un número menor, por lo cual el número final de retuits recolectados fue de 114777 .

7 Twelets es un software en línea que permite recolectar tuits, favoritos, retuits y seguidores directamente de las cuentas. El programa puede ser utilizado en la siguiente dirección http://twlets.com/

8 AntConc es un paquete de software independiente para el análisis lingüístico de textos, libremente disponible para Windows, macOS y Linux, que permite trabajar en un PDF o un documento de Word 25 http://www.laurenceanthony.net/software/antconc/ 
de estas. Con este fin, se analizó el corpus de las cuentas del Centro Democrático utilizando la herramienta AntConc. En primer lugar, se extrajeron las palabras que presentan alta frecuencia dentro del corpus estudiado. En segundo lugar, con el mismo software, se hallaron algunas keywords ${ }^{9}$ entre el corpus de tuits de los congresistas del Centro Democrático respecto del corpus de las cuentas de Álvaro Uribe y del partido (Rayson y Garside, 2000). Así, mediante el coeficiente de keyness, ${ }^{10}$ se buscó establecer hasta qué punto la agenda temática del Centro Democrático es compartida por los congresistas de esta colectividad.

En la segunda fase, por medio de correlaciones de Pearson, se estimaron asociaciones entre las palabras más utilizadas en el corpus de los congresistas en Twitter. Esta estrategia se utilizó para identificar patrones entre las palabras más utilizadas y, de esta manera, explorar cómo se articula la agenda heterogénea u homogénea del Centro Democrático en Twitter: si está orientada hacia una estrategia del endogrupo y exogrupo o si sigue patrones personalistas. Finalmente, se presentaron y discutieron estos resultados según la evidencia empírica.

\section{Resultados}

Para buscar si existe una homogeneidad en la agenda temática del Centro Democrático o no, se siguió un proceso en dos etapas (Bolívar, 2016; Mickiewicz, 2015). En primer lugar, se estableció un gran corpus para describir las principales palabras utilizadas en las cuentas de Twitter. Posteriormente, utilizando el freeware AntConc, se escogieron las palabras más repetidas para hacer la interpretación de los datos obtenidos a partir de tuits elaborados y circulados a través de esta red social para testear las hipótesis propuestas (Glynn y Fischer, 2010). Las repeticiones de las palabras encontradas puede ser una de las maneras en que el partido las dota de significado para homogeneizar su propia agenda temática (Bauman y May, 2001) (figura 1).

9 Palabras y frases que son significativamente más frecuentes en un corpus determinado frente a uno de referencia.

10 Indica en qué grado una palabra aparece con una frecuencia inusualmente alta en relación con un corpus de referencia (Bondi y Scott, 2010, pp. 3-4). 


\section{Figura 1. Palabras más frecuentes en las cuentas de Twitter del Centro Democrático}

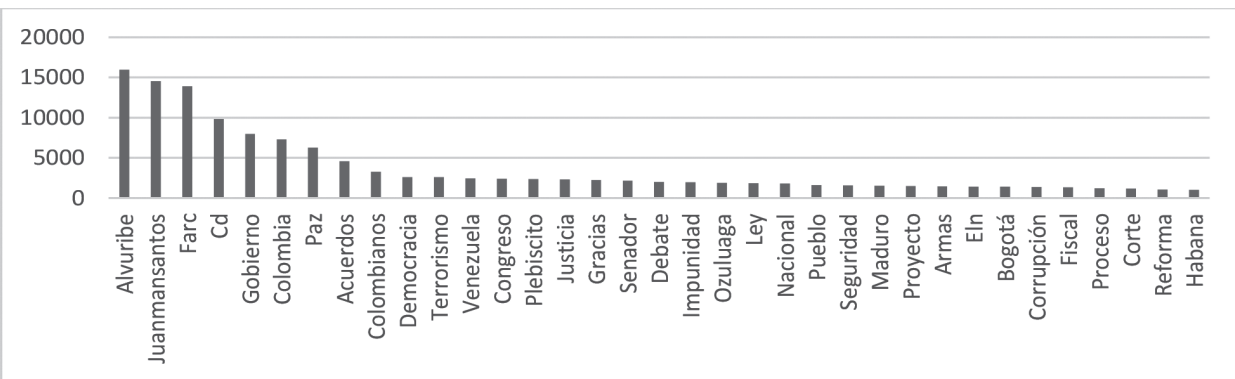

Fuente: elaboración propia.

Dentro de las palabras más frecuentes en el corpus de cuentas de Twitter del Centro Democrático, se encontraron, en primer lugar, las referencias al líder del partido, el senador Álvaro Uribe, seguido de los dos antagonistas de este movimiento político, el presidente Juan Manuel Santos y las FARC y, finalmente, el Centro Democrático. Esto parece corresponderse a un uso sistemático de estas palabras para identificar un endogrupo y un exogrupo y en la presencia de una agenda temática homogénea, como se esperaba desde la teoría de la identidad social. Se denota que hay una uniformidad en el reconocimiento de los principales antagonistas FARC y Juan Manuel Santos (ellos) y una identificación de un nosotros en torno al expresidente Álvaro Uribe y, de manera secundaria, al partido.

Una vez identificadas las palabras más repetidas, se procedió a testear las hipótesis de investigación por medio de la estimación de un análisis de concordancia. Para tal objetivo, se utilizó el freeware AntConc. Este permitió generar siete keywords, estas son elementos léxicos con altas frecuencias relativas, o keyness, lo cual indica que estas palabras son relevantes respecto de la probabilidad estadística de su aparición, y, por tanto, proporcionan información sobre la concordancia temática de las distintas cuentas con las del partido y su líder (Scott y Tribble, 2006). De tal forma, se puede considerar estas keywords para evaluar si la agenda temática del partido es homogénea o heterogénea, frente a las dos cuentas que se tomaron como referentes. 


\section{Tabla 1. Keywords del discurso de los senadores del Centro Democrático frente al de Álvaro Uribe}

\begin{tabular}{|c|c|c|}
\hline Keyword & Frecuencia & keyness \\
\hline alvarouribevel & 13317 & 334761 \\
\hline cedemocratico & 7523 & 66682 \\
\hline victimas & 847 & 44665 \\
\hline oizuluaga & 1886 & 44490 \\
\hline paz & 6175 & 43652 \\
\hline acuerdos & 1592 & 38420 \\
\hline habana & 1010 & 26642 \\
\hline
\end{tabular}

Fuente: elaboración propia.

Las keywords no siempre coinciden con aquellas que tienen mayor frecuencia en el corpus. En la tabla 1, se encuentra que "victimas" está en tercer lugar, pese a que su frecuencia es baja, esto se debe a que el nivel de keyness, es particularmente alto. Mediante este análisis se observa cuáles son las palabras que se repiten con frecuencia inusual en el corpus de las cuentas de los miembros del Centro Democrático con referencia a la cuenta del líder del partido, y qué puede decir esto sobre la homogeneidad de la agenda temática en Twitter.

Asimismo, en la tabla 1, se puede observar la lista de palabras que tienen un keyness sobre 25000 . En general, este nivel es bajo dentro de la muestra referenciada, lo cual indica que no hay una diferencia importante entre el discurso de Álvaro Uribe en Twitter y las cuentas de los demás congresistas del Centro Democrático. Esta uniformidad se refuerza al ver que la keyword más importante, y de manera considerable, es la misma cuenta del líder del partido. Ahora, en segundo lugar, y por una distancia importante, aparece la cuenta oficial del Centro Democrático; esto indica que, en general, los congresistas de este partido, desde sus cuentas de Twitter, retuitean o mencionan en sus tuits las publicaciones hechas por el líder de la colectividad y de la cuenta del partido. De esta manera, se evidencia un comportamiento en general homogéneo y alineado al discurso del líder (Mustafaraj et al., 2011; Conover, Gonçalves, Flammini y Menczer, 2012; Himelboim, McCreery y Smith, 2013). Además, las palabras que tienen un menor key- 
ness son indicio de que hay temas que los congresistas visibilizan más, con referencia a Álvaro Uribe, pero que no presentan una desviación importante, es decir que se encuentran dentro de la agenda del líder.

\section{Tabla 2. Keywords del discurso de los senadores del Centro Democrático frente a la cuenta del partido}

\begin{tabular}{|c|c|c|}
\hline Palabra & Frecuencia & Keyness \\
\hline abril & 2417 & 95999 \\
\hline plebiscito & 2331 & 68273 \\
\hline marcha & 1210 & 50462 \\
\hline victimas & 847 & 49858 \\
\hline oderbrecht & 881 & 37665 \\
\hline saludhernandezm & 838 & 35334 \\
\hline noticiasrcn & 1077 & 35053 \\
\hline
\end{tabular}

Fuente: elaboración propia.

Ahora, en cuanto a la comparación del corpus de las cuentas de los congresistas con la del partido, en la tabla 2 , se puede observar que en general el keyness es bajo, lo cual indica que tampoco hay una diferencia importante entre el discurso de los congresistas del Centro Democrático con su partido. En este análisis, vuelve a aparecer la palabra "victimas", lo cual indica que los congresistas hablan más de ellas que lo que lo hace tanto el partido como Álvaro Uribe, cuyas cuentas hablan sobre víctimas, pero no de la misma manera con que lo hacen los congresistas del Centro Democrático. Esta recurrencia y el bajo nivel de keyness dentro de la muestra pueden mostrar una ligera desviación del discurso de los congresistas frente al de su líder y la cuenta del partido, que, si bien refleja una visibilización de otros temas, no se aleja de su agenda.

Por otro lado, las tres primeras palabras ("abril”, "plebiscito" y "marcha") hacen referencia a hechos específicos en los que la colectividad estuvo unida y actuó de manera conjunta: el plebiscito del 2 de octubre de 2016 y la marcha del $1^{\circ}$ de abril de 2017. Los congresistas actuaron en concordancia con la agenda partidista, superando, al menos en el discurso, la visibilización de estos dos momentos, frente a lo que hizo el partido desde 
su cuenta de Twitter y así mostró una homogeneidad en la agenda del partido frente a momentos específicos en los que la colectividad se presentó unida. Por último, la aparición de las cuentas de la periodista española Salud Hernández y de noticias RCN en la tabla 2 indican que los congresistas del Centro Democrático acuden recurrentemente a retuitear las opiniones de la comunicadora y las noticias que publica esta cadena colombiana, ya que, posiblemente, se pueden sentir identificados con la ideología expresada en sus publicaciones en esta red social.

Este análisis estadístico permite aportar evidencia empírica para confirmar la segunda hipótesis, en la medida en que los miembros del Centro Democrático, según el análisis de concordancia a partir de la identificación de keywords, tienen una agenda relativamente homogénea. De igual forma, las palabras más recurrentes y comunes en todos los congresistas tienen relación con el endogrupo y exogrupo, por lo cual se puede afirmar que hay una estrategia de identificación social según el reconocimiento y la atribución de adjetivos a un rival externo. No obstante, los congresistas también manejan una agenda propia que, si bien es cierto no se aleja de la del partido, sí muestra algunos intereses personales por visibilizar algunos temas.

\section{La homogeneidad temática y la identificación social}

El análisis de concordancia permitió confirmar la segunda hipótesis de la agenda temática homogénea en el corpus de las cuentas del Centro Democrático. En esta segunda sección, se explora la forma en que se identifican los endogrupos y exogrupos por parte de los congresistas de este partido siguiendo el esquema planteado por Adrián (2013). Según este objetivo, se buscó establecer las asociaciones entre las palabras identificadas. Para evaluar la existencia de asociación en promedio en la población, entre las palabras se realizaron correlaciones de Pearson donde la hipótesis nula fue establecida favoreciendo una asociación de cero $(H 0: \mu$ asociación $=0)$ y una hipótesis alternativa favoreciendo una asociación diferente de cero ( Hi: $\mu$ asociación $\neq 0$ ). Los resultados de esta prueba de hipótesis aportan evidencia para rechazar la hipótesis nula con un valor alpha de 0.05 y así 
establecer las palabras que tienen un mayor número de correlaciones significativas y positivas con otras.

Estas estimaciones permiten conocer cuáles palabras están asociadas significativamente con otras en el corpus de tuits de los congresistas del Centro Democrático y así explorar a cuáles palabras se le adjudican un mayor o menor número de atributos positivos y negativos. Si bien el número de correlaciones no permite distinguir entre el exogrupo y el endogrupo, sí hace posible observar cuáles están más asociadas con otros adjetivos y temas.

En la figura 2, se observa que las palabras con mayor número de asociaciones son "FARC” (18), "Juan Manuel Santos" (17), "gobierno” (16) y "justicia” (16). Al tiempo que "Álvaro Uribe" (3) y "Centro Democrático" (3) tienen un menor número de asociaciones, a pesar de ser las más repetidas en el corpus.

\section{Figura 2. Número de asociaciones por palabra}

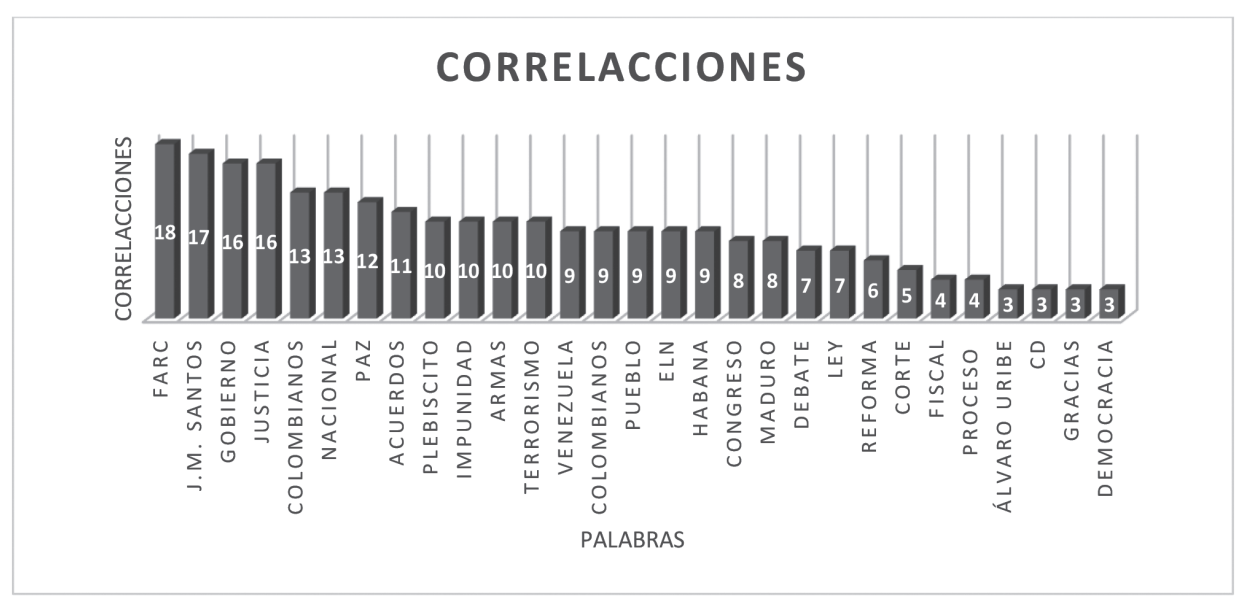

Fuente: elaboración propia.

Estos hallazgos aportan evidencia empírica que sugiere que la mayor actividad en Twitter está enfocada en asociar rasgos al exogrupo, mientras que no se asocia un gran número de palabras al endogrupo, por lo cual se privilegia más una estrategia en asignar valores compartidos a los antagonistas. En tanto que, en torno al endogrupo, se sigue una lógica más auto- 
rreferencial. Esto es, la frecuencia de repetición de palabras que se refieren al endogrupo ("Álvaro Uribe”, "Centro Democrático") es constante, pero ellas no están asociadas a un gran número de palabras negativas o positivas.

A continuación, se analizan las palabras con mayor número de correlaciones para ver si, en efecto, estas responden a una estrategia de construir relaciones entre palabras negativas y los antagonistas políticos. Para tal fin, por un lado, se analizaron a fondo las dos palabras que hacen referencia a los principales antagonistas de este partido político y que muestran un mayor número de correlaciones; y por otro, las palabras que utilizan los congresistas del Centro Democrático para identificar su colectividad.

\section{Figura 3. Número de asociaciones por palabra con "FARC"}

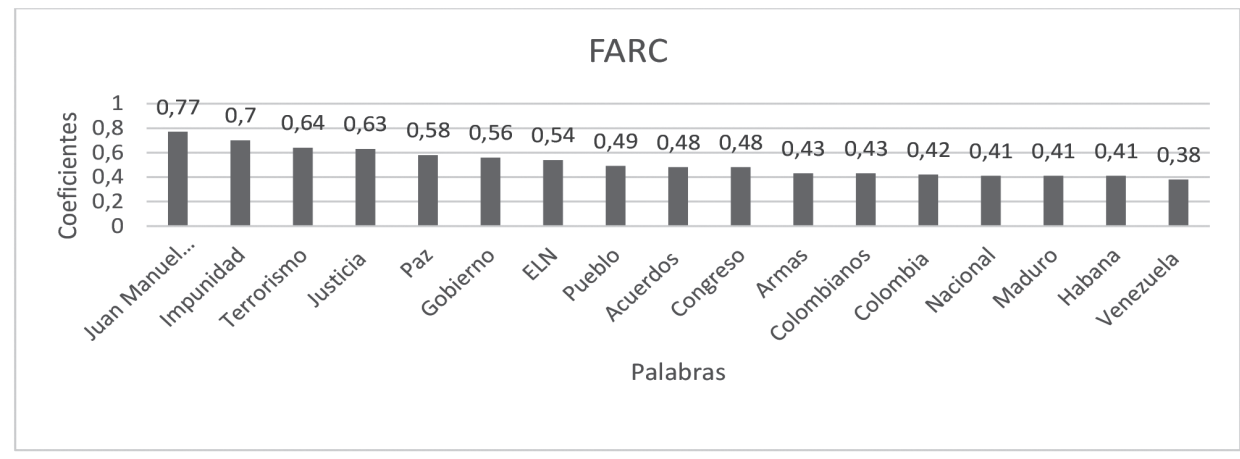

Fuente: elaboración propia

En la figura 3, se observa que, entre las palabras que están relacionadas con la palabra "FARC", sobresale la asociación con "Juan Manuel Santos", que tiene un coeficiente de 0.77 , lo cual indica que en la mayoría de las ocasiones en que en las cuentas escriben la palabra "FARC" también mencionan el nombre del presidente. Por consiguiente, se intenta relacionar la palabra "Juan Manuel Santos" con esta organización. Asimismo, los coeficientes de "terrorismo" e "impunidad" también son altos, lo cual permitiría suponer que la palabra "FARC" en la mayoría de las ocasiones es reseñada junto a la de "impunidad" y "terrorismo". Las siguientes palabras: "justicia", "paz" y "gobierno" son más difíciles de interpretar en la medida en que el análisis elaborado en este artículo carece de la interpretación del contexto 
discursivo donde se enuncian. Se puede suponer que pueden ser utilizadas negativamente, como necesidad de más justicia respecto de este grupo, falsa paz o aliados del Gobierno. No obstante, se necesitaría una aproximación cualitativa para sustentar estas afirmaciones, lo cual escapa de los alcances y objetivos de este artículo. Las investigaciones de corte cualitativo (Velasco, 2017; Caicedo, 2016) han mostrado que estas palabras positivas se utilizan negativamente como con la expresión “iesta es la paz de Santos?”, por lo cual no se subrayaría el contenido positivo, sino una relación negativa entre las "FARC” y "menos paz" o "necesidad de más justicia”. Finalmente, palabras como "Maduro", "Venezuela” y "Habana” reflejan la estrategia de asociar estos actores con las FARC, especialmente en torno al tema de la paz y la negociación de los acuerdos con el Gobierno.

\section{Figura 4. Número de asociaciones por palabra con "Juan Manuel Santos"}

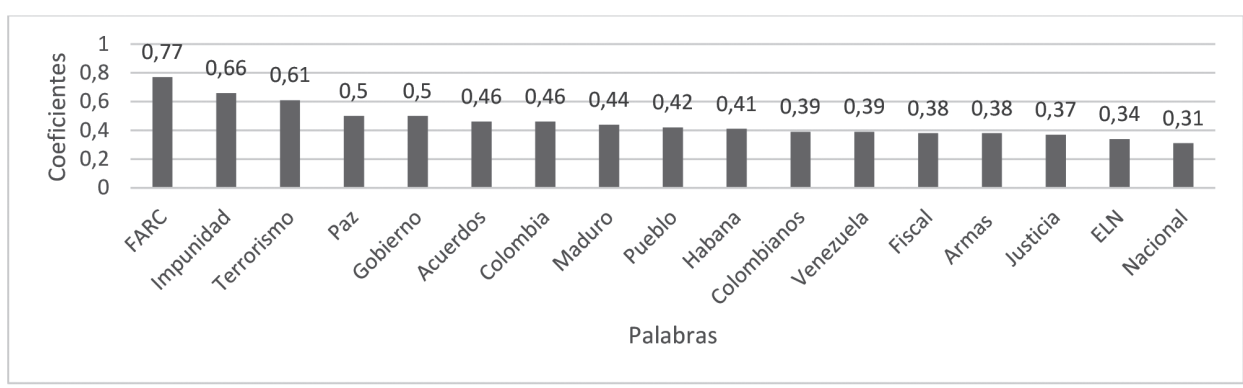

Fuente: elaboración propia.

En la figura 4, se observan las palabras que están relacionadas con la palabra "Juan Manuel Santos” y los coeficientes de correlación. En la comparación entre las dos figuras, se puede observar que se realiza un proceso de construcción de antagonistas muy similar al descrito. La diferencia más importante es que el tema de justicia está más relacionado con las "FARC" que con "Juan Manuel Santos”, pero, igual, el nombre del presidente está asociado a las palabras "impunidad", "terrorismo" y "paz”. Esto puede evidenciar la intención de asociar la paz, que se ha constituido en el principal tema del Gobierno, con los aspectos negativos que este partido ha venido criticando a lo largo del periodo presidencial. También se presenta una asociación del presidente con las palabras "Maduro", "Habana” y "Venezuela”, 
lo cual se corresponde con la estrategia de relacionar estas palabras con la del presidente Juan Manuel Santos.

\section{Figura 5. Número de asociaciones por palabra con "Álvaro Uribe"}

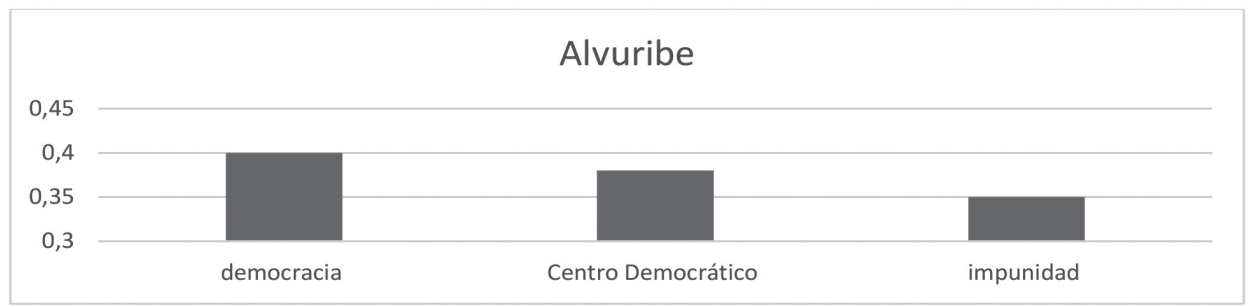

Fuente: elaboración propia.

Además de las correlaciones existentes de los antagonistas del partido, se analizaron las existentes con las cuentas de Álvaro Uribe y del Centro Democrático para explorar si, en efecto, la agenda del partido responde a una lógica de autorreferenciación con el objetivo de construir una identidad partidista. Pese a que forman parte de las palabras más repetidas dentro del corpus, ellas no están asociadas a un gran número de palabras negativas o positivas. Un aspecto para destacar es que en ambas correlaciones aparece la palabra "democracia", esto no es de sorprender, ya que, en el discurso político, esta tiene un valor polisémico y permite describir muchas cosas sin una conexión directa con la realidad. Es decir que es una palabra que es de fácil manipulación para los intereses de los partidos y los políticos (Wodak y Koller, 2008; De Saussure y Schulz, 2005; Chilton, 2002; Ascanio, 2001; Perez Huggins, 1982). El uso de esta palabra puede hacerse para sostener la propia ideología, denunciar algún atentado contra ella, denunciar alguna agresión o para atacar (Connolly, 1993; Gallie, 1955). Tal podría suponerse que es la estrategia de los congresistas del Centro Democrático, quienes buscan establecer valores positivos para el endogrupo, puede ser como denunciantes ante falta de democracia $o$ atentados contra ella, mientras que se asignan valores negativos al exogrupo como agente que atenta contra su buen ejercicio, sobre todo en cuanto a la firma del Tratado de Paz de La Habana y su refrendación popular (Semana, 2016; El Tiempo, 2016). 
En la figura 5, se puede ver que las palabras que están relacionadas con "Álvaro Uribe”, además de “democracia”, se encuentra la mención al partido, lo cual indica que se busca asociar la figura del expresidente a la colectividad, siguiendo la estrategia de autorreferenciación y posicionando a Uribe como uno de los personajes más importantes del partido. Por otro lado, la correlación con la palabra "impunidad" es difícil de analizar en cuanto no se cuenta con el contexto discursivo de enunciación; sin embargo, se puede suponer que se hace referencia al senador Uribe como denunciante de casos de la impunidad que puede generar el tratado de paz.

\section{Figura 6. Número de asociaciones por palabra con "Centro Democrático"}

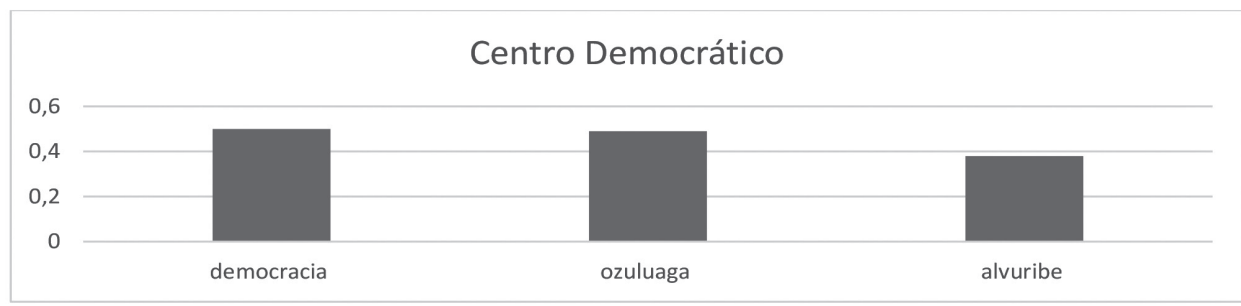

Fuente: elaboración propia.

Por último, en la figura 6, se observa que, además de "democracia”, las palabras con mayor coeficiente de correlación con el Centro Democrático hacen referencia a Óscar Iván Zuluaga (expresidente del partido) y a Álvaro Uribe. Este comportamiento refuerza la idea de que los miembros del partido siguen una estrategia de autorreferenciación, identificando el partido con sus líderes principales.

El comportamiento en Twitter corresponde a lo evidenciado en otras investigaciones (López, 2016; Osborne y Sibley, 2015; Adrián, 2013), donde se señala que los partidos utilizan estrategias de constitución de endogrupo y exogrupo atribuyendo rasgos positivos al primero y negativos al segundo. En el caso del Centro Democrático, se demostró que este partido privilegia la estrategia del exogrupo relacionándolo con una amplia gama de adjetivos y palabras consideradas negativas como "terrorismo" e "impunidad". 


\section{Discusión y conclusiones}

Los resultados obtenidos a partir del análisis del corpus del Centro Democrático se corresponden con las expectativas teóricas de la teoría de la identidad social más que del personalismo político. Los congresistas del Centro Democrático no son propensos a asumir agendas temáticas propias e independientes, sino a tener una agenda colectiva relativamente homogénea. A pesar de que diversas investigaciones han resaltado los comportamientos individualistas de los políticos colombianos frente a sus partidos y su escasa diferenciación temática (Correa y Camargo, 2017; García y Hoskin, 2006), el estudio actual muestra que los congresistas en Twitter tienen una agenda homogénea que se distingue, especialmente por una estrategia de identificación de dos antagonistas principales, las FARC y el presidente Juan Manuel Santos.

Los datos y este tipo de estudio son novedosos e intentaron ser exhaustivos, pero esta investigación tiene ciertas limitaciones. En primer lugar, el estudio se centró en el caso del Centro Democrático, lo cual es una restricción en la medida en que no permite la generalización de los resultados evidenciados a políticos de otros partidos, más aún por el carácter sui generis de esta colectividad dentro del sistema de partidos colombianos. El Centro Democrático fue uno de los pocos partidos que postuló listas cerradas $^{11}$ al Congreso en las elecciones de 2014 y se caracterizó por la fuerte influencia de su líder, Álvaro Uribe. No obstante, esta aproximación permitió no solo explorar la forma en que los políticos están definiendo sus posturas en Twitter, sino identificar también los patrones colectivos en esta red social de los miembros de esta agrupación política. En una próxima investigación, la muestra se puede ampliar a otros partidos, lo cual permitiría una mayor generalización de estos resultados. Los hallazgos también respaldan de forma inicial las expectativas de la teoría de la identidad social y es posible que esta pueda ser utilizada para aumentar la compresión del comportamiento de los políticos en Twitter, aun en el caso de otros partidos políticos menos cohesionados respecto de su líder.

11 Una de las características del sistema electoral colombiano es que los partidos pueden presentar listas abiertas o cerradas para elegir cargos plurinominales, como los concejos, las asambleas y el Congreso. En las listas cerradas, los votantes pueden escoger el partido y el candidato por el cual quieren votar, mientras que, en el caso de las listas cerradas, solo se vota por el partido y es este el que establece el orden de inscripción de sus candidatos. 
Las investigaciones en comunicación política han señalado el aumento de las tácticas de ataque de los políticos a sus contradictores en Twitter (Sapiro, Hemphill y Otterbacher, 2012). Los resultados de esta investigación reflejan que, en los mensajes en Twitter, los congresistas del Centro Democrático también siguen esta estrategia. Los análisis en este estudio sugieren que los congresistas, más que enfocarse en ellos mismos o en sus temas particulares, utilizan las redes sociales para dirigir su uso a la identificación y el ataque de antagonistas del partido. Las futuras investigaciones deberían continuar explorando estas tendencias y comparar las distintas agendas que los políticos tienen en Twitter para determinar el alcance y la naturaleza del uso de esta red social en la comunicación política de los partidos.

La existencia de una agenda temática homogénea en Twitter haría más fácil a los ciudadanos identificar esta colectividad y sus principales posturas (Knowles et al., 2013). Por lo cual, este partido puede ser un comunicador efectivo en sus mensajes e interacción en esta red social para comunicárselos a sus seguidores y promover una votación más programática que partidos con una agenda temática más heterogénea.

En conclusión, el artículo hace contribuciones teóricas que permiten avanzar en la comprensión de la forma en que interactúan los partidos y políticos en Twitter. Asimismo, propone herramientas metodológicas para explorar e identificar la homogeneidad o heterogeneidad de las agendas de los partidos políticos en esta red social. El análisis utilizado en este artículo permitirá a futuras investigaciones realizar comparaciones entre agendas temáticas de distintos partidos políticos y analizar las estrategias a través del contenido de sus tuits. Finalmente, el análisis permite identificar la forma en que el Centro Democrático ha utilizado Twitter y da indicios de las estrategias que ha empleado este partido para comunicarse con sus audiencias virtuales.

\section{Agradecimientos}

Los autores agradecen a Sebastián Pantoja, Sergio Barbosa y Carlos Toro, así como a dos revisores anónimos, por sus comentarios y sugerencias. También, a Lorena Pardo por su colaboración en las etapas preliminares de este proyecto. 


\section{Referencias}

Abramowitz, A. y Webster S. (2015). All politics is national: The rise of negative partisanship and the nationalization of US house and senate elections in the 21st century. Trabajo presentado en Annual Meeting of the Midwest Political Science Association, Chicago.

Adi, A., Erickson, K. y Lilleker, D. G. (2014). Elite tweets: Analyzing the Twitter communication patterns of Labour party peers in the House of Lords. Policy \& Internet, 6(1), 1-27. DOI: 10.1002/19442866.POI350

Adrián, T. (2013). Sinonimia y oposiciones léxico semánticas en discursos de Hugo Chávez: un estudio político ideológico de Hugo Chávez Frías. Revista Latinoamericana de Estudios del Discurso, 13(1), 9-32.

Aharony, N. (2012). Twitter use by three political leaders: An exploratory analysis. Online Information Review, 36(4), 587-603. https://doi. org/10.1108/14684521211254086

Alhabash, S. y McAlister, A. R. (2014). Redefining virality in less broad strokes: Predicting viral behavioral intentions from motivations and uses of Facebook and Twitter. New Media \& Society, 17(8), 1317-1339.

Ascanio, A. (2001). Análisis del contenido del discurso político. Caracas: Equinoccio.

Bafumi, J. y Shapiro, R. Y. (2009). A new partisan voter. The Journal of Politics, 71(1), 1-24.https://doi.org/10.1017/S0022381608090014

Bauman, Z. y May, T. (2001). Thinking sociologically (2. a ed.). Oxford: Wiley-Blackwell.

Becerra, J. (2015). Las redes sociales en internet: una nueva forma de comunicación y socialización. México: Universidad Nacional Autónoma de México. 
Bolívar, A. (2016). La definición del corpus en los estudios del discurso. Revista Latinoamericana de Estudios del Discurso, 13(1), 3-8.

Bondi, M. y Scott, M. (eds.) (2010). Keyness in texts. Ámsterdam: John Benjamins Pub. Co.

Botero, F. (ed.). (2011). Partidos y elecciones en Colombia. Bogotá: Universidad de los Andes.

Caicedo,J.M. (2016). “¿Esta es la paz de Santos?”: el partido Centro Democrático y su construcción de significados alrededor de las negociaciones de paz. Revista CS, 19, 15-37.http://dx.doi.org/10.18046/ recs.i19.2136

Carey, J. M. y Shugart, M. S. (1995). Incentives to cultivate a personal vote: A rank ordering of electoral formulas. Electoral Studies, 14(4), 417439. https://doi.org/10.1016/0261-3794(94)00035-2

Charaudeau, P. y Mainguenau, D. (2002). Dictionnaire d'analyse du discours. París: Éditions du Seuil.

Chilton, P. (2002). Political terminology. En R. Wodak y V. Koller (eds.), Handbook of communication in the public sphere (vol. 4, pp. 226-42). Berlín: Walter de Gruyter GmbH \& Co.

Cogburn, D. L. y Espinoza-Vasquez, F. K. (2011). From networked nominee to networked nation: Examining the impact of Web 2.0 and social media on political participation and civic engagement in the 2008 Obama campaign. Journal of Political Marketing, 10(12), 189-213. https://doi.org/10.1080/15377857.2011.540224

Connolly, W. E. (1993). The terms of political discourse. Princeton, N.J: Princeton University Press.

Conover, M. D., Gonçalves, B., Flammini, A. y Menczer, F. (2012). Partisan asymmetries in online political activity. EPJ Data Science, 1(6). doi:10.1140/epjds6 
Conway, B. A., Kenski, K. y Wang, D. (2013). Twitter use by presidential primary candidates during the 2012 campaign. American Behavioral Scientist, 57(11), 1596-1610. https://doi. org/10.1177/0002764213489014

Correa, J. C.y Camargo, J.E. (2017). Ideological consumerism in colombian elections, 2015: Links between political ideology, Twitter activity, and electoral results. Cyberpsychology, Behavior, and Social Networking, 20(1),37-43. https://doi.org/10.1089/cyber.2016.0402

Dosek, T. y Freidenberg, F. (2014). Voto hacia nuevos candidatos en sistemas de partidos (des) institucionalizados: el caso de Marco Enríquez-Ominami en Chile 2009. Revista SAAP, 8(1), 11-42.

Duque, J. (2011). El Congreso de la República en Colombia: estabilidad y renovación en las elecciones de 2010. Colombia Internacional, 74, 119-151.

Edwards, M. S. y Thames, F. C. (2007). District magnitude, personal votes, and government expenditures. Electoral Studies, 26(2), 338-345. https:// doi.org/10.1016/j.electstud.2006.06.008

El Tiempo (2016, agosto 27). "La verdadera paz empieza con el No". Recuperado de http://www.eltiempo.com/especiales/argumentos-del-centro-democratico-para-votar-no-en-el-plebiscitopor-la-paz-42163

Gallie, W. B. (1955). Essentially contested concepts. Proceedings of the Aristotelian Society, 56, 167-198.

García, M. y Hoskin, G. (2006). Reforma política, personalismo y sistema de partidos: ¿partidos fuertes o coaliciones electorales? En G. Hoskin y M. García Sánchez (comps.), La reforma política de 2003: ¿la salvación de los partidos políticos colombianos? (pp. 139-159). Bogotá: Universidad de los Andes. 
García, M. y Wills, L. (2011). El poder de la televisión: medios de comunicación y aprobación presidencial en Colombia. En A. Rettberg y O. Rincón (comps), Medios, democracia y poder: una mirada comparada desde Colombia, Ecuador, Venezuela y Argentina (pp. 135157). Bogotá: Universidad de los Andes.

Gayo, D., Metaxas, P. T.y Mustafaraj, E. (2011). Limits of electoral predictions using twitter. Ponencia presentada en Proceedings of the Fifth International AAAI Conference on Weblogs and Social Media, Association for the Advancement of Artificial Intelligence.

Glynn, D. y Fischer, K. (2010). Quantitative methods in cognitive semantics: Corpus-driven approaches. Berlín: Walter de Gruyter.

Grussel, M. y Nord, L. (2012). Three attitudes to 140 characters: The use and views of Twitter in political party communications in Sweden. Public Communication Review, 2(2), 48-61.

Gunther, R. y Diamond, L. (2003). Species of political parties: A new typology. Party Politics, 9(2), 167-199.

Gutiérrez, F. (2007). ¿Lo que el viento se llevó? Los partidos políticos y la democracia en Colombia, 1958-2002. Bogotá: Norma.

Hanson, G., Haridakis, P. M., Cunningham, A. W., Sharma, R. y Ponder, J. D. (2010). The 2008 presidential campaign: Political cynicism in the age of Facebook, MySpace, and YouTube. Mass Communication and Society, 13(5), 584-607. https://doi.org/10.1080/1520 5436.2010 .513470

Himelboim, I., McCreery, S. y Smith, M. (2013). Birds of a feather tuit together: Integrating network and content analyses to examine cross-ideology exposure on Twitter. Journal of Computer-Mediated Communication, 18(2), 40-60. DOI: $10.1111 /$ jcc4.12001 
Hoskin, G. y García, M. (comps.) (2006). La reforma política de 2003: ¿la salvación de los partidos políticos colombianos? Bogotá: Universidad de los Andes.

Issacharoff, S. (2016). Outsourcing politics: The hostile takeovers of our hollowed out political parties. Recuperado de https://papers.ssrn. com/sol3/papers.cfm?abstract_id $=2888064$

Iyengar, S., Sood, G. y Lelkes, Y. (2012). Affect, not ideology: A social identity perspective on polarization. Public Opinion Quarterly, 76(3), 405-431. https://doi.org/10.1093/poq/nfs038

Kamasa, V. (2015). Corpus linguistics for critical discourse analysis: What can we do better? Recuperado de https://goo.gl/XSHrcK

Kenski, K., Filer, C. R. y Conway, B. A. (2017). Communicating party labels and names on Twitter during the 2016 presidential invisible primary and primary campaigns. Journal of Political Marketing, 16(34), 267-289. https://doi.org/10.1080/15377857.2017.1334253

Knowles, E. D., Lowery, B. S., Shulman, E. P. y Schaumberg, R. L. (2013). Race, ideology, and the Tea Party: A longitudinal study. PLoS ONE, 8(6), e67110. https://doi.org/10.1371/journal.pone.0067110

Kruikemeier, S. (2014). How political candidates use Twitter and the impact on votes. Computers in Human Behavior, 34, 131-139. https:// doi.org/http://dx.doi.org/10.1016/j.chb.2014.01.025

Lancaster, T. D. (1986). Electoral structures and pork barrel politics. International Political Science Review, 7(1), 67-81.

López, J. L. (2016). La activación de identidades: la redefinición de endogrupos y exogrupos. Télos, 20(2), 105-125.

Losada, R. yLiendo, N. (2016). El partido Centro Democrático en Colombia: razones de su surgimiento y éxito. Análisis Político, 29(87), 41-59. 
Luhmann, N. (1990). Sociedad y sistema: la ambición de la teoría. Madrid: Paidós.

Mainwaring, S. y Bejarano, A. M. (eds.) (2008). La crisis de representación democrática en los países andinos. Bogotá: Norma.

Mainwaring, S. y Scully, T. (eds.) (1995). Building democratic institutions: Party systems in Latin America. Stanford, CA: Stanford University Press.

Milanese,J. P., Abadía, A. A. y Manfredi, L. (2016). Elecciones regionales en Colombia 2015: análisis de las nuevas gobernaciones y asambleas departamentales. Revista Uruguaya de Ciencia Política, 25(1), 1-26.

Norza-Céspedes, E. y Espino-Duque, G. P. (2011). Criminalidad y análisis espacial de los delitos en Colombia, 2010. Revista Criminalidad, 53(1), 17-43.

Norza, E., Peñalosa, M. J. y Rodríguez, J. (2017). Exégesis de los registros de criminalidad y actividad operativa de la Policía Nacional, año 2016 en Colombia. Revista Criminalidad, 59(2), 15-29.

Novaes, L. M. (2015). Modular parties: Party systems with detachable clienteles (Tesis doctoral, University of California, Berkeley, Estados Unidos). Recuperado dehttp://digitalassets.lib.berkeley.edu/etd/ ucb/text/Novaes_berkeley_0028E_15846.pdf

Osborne, D. y Sibley, C. G. (2015). Opposing paths to ideology: Groupbased relative deprivation predicts conservatism through warmth toward ingroup and outgroup members. Social Justice Research, 28(1), 27-51. https://doi.org/10.1007/s11211-014-0227-1

Pérez Huggins, A. (1982). Betancourt y Caldera: discurso e ideología. Caracas: Universidad Central de Venezuela. 
Persily, N. y Cain, B. E. (2000). The legal status of political parties: A reassessment of competing paradigms. Columbia Law Review, 100(3). https://doi.org/10.2307/1123502

Peterson, R. D. (2012). To tweet or not to tweet: Exploring the determinants of early adoption of Twitter by House members in the 111th Congress. The Social Science Journal, 49(4), 430-438. https:// doi. org/10.1016/j.soscij.2012.07.002

Pino, J. F. (2017). Entre democracias y autoritarismos: una mirada crítica al estudio de la democracia subnacional en Colombia y Latinoamérica. Colombia Internacional, 91, 215-242. https://doi.org/10.7440/ colombiaint91.2017.07

Pizarro, E. (2008). Gigantes con pies de barro. En A. M. Bejarano y S. Mainwaring (eds.), La crisis de representación democrática en los países andinos. Bogotá: Norma.

Ramírez-Castro, J. (2014). Experiences and expectations in political debate: Young people in Manizales and the elections. Revista Latinoamericana de Ciencias Sociales, Niñez y Juventud, 12(1), 243-256. https://doi.org/10.11600/1692715x.12114081313

Rayson, P.y Garside, R. (2000). Comparing corpora using frequency profiling. Ponencia presentada en Proceedings of the workshop on Comparing Corpora, Hong Kong, Association for Computational Linguistics. https://doi.org/10.3115/1117729.1117730

Resimić, M. (2016). Media coverage of violence against women in the family and in an intimate partner relationship in Serbia. Temida, 19(1), 63-82. https://doi.org/10.2298/TEM1601063R

Rincón, O. (2011). Mucho ciberactivismo... pocos votos: Antanas Mockus y el Partido Verde colombiano. Nueva Sociedad, 235, 74-89. 
Ross, K.y Bürger, T. (2014). Face to face(book): Social media, political campaigning and the unbearable lightness of being there. Political Science, 66(1), 46-62. https://doi.org/10.1177/0032318714534106

Roth, M., Peters, G. y Seruga, J. (2014). Tweeting politicians: An analysis of the usage of a micro blogging system. En S. Hammoudi, J. Cordeiro, L. A. Maciaszek y J. Filipe (eds.), Enterprise information systems (vol. 190). Cham: Springer International Publishing. https:// doi.org/10.1007/978-3-319-09492-2

Santamaría, N. (2013). Determinantes de la conformación del quórum en el Congreso Colombiano: evidencia a partir de las votaciones nominales. Documentos de Ciencia Política, 24, 1-23.

Sapiro M. A., Hemphill, L.y Otterbacher, J. (2012). Going 'Bald on Record': Relationships among public officials' social media behavior and language use. Ponencia presentada en MPSA Conference, Chicago, IL.

Sartori, G. (1980). Partidos y sistemas de partidos. Madrid: Alianza.

Saussure, L. de y Schulz, P. (eds.) (2005). Manipulation and ideologies in the twentieth century: Discourse, language, mind. Ámsterdam: John Benjamins Publishing.

Scott, M. y Tribble, C. (2006). Textual patterns: Key words and corpus analysis in language education. Ámsterdam: John Benjamins Publishing.

Semana (2016, mayo 12). "Es un golpe de Estado a la democracia": uribismo. Recuperado de http://www.semana.com/nacion/articulo/ partido-de-alvaro-uribe-rechaza-acuerdo-entre-farc-y-gobiernopara-blindar-acuerdos-de-paz/473325

Shugart, M. S. y Pachón, M. (2010). Electoral reform and the mirror image of inter-party and intra-party competition: The adoption of party lists in Colombia. Electoral Studies, 29(4), 648-660. 
Sinclair, J. (1991). Corpus, concordance, collocation. Oxford: Oxford University Press.

Šķilters, J., Kreile, M., Bojārs, U., Brikše, I., Pencis, J. y Uzule, L. (2012). The pragmatics of political messages in Twitter communication. En R. García-Castro, D. Fensel y G. Antoniou (eds.), The Semantic Web: ESWC 2011 Workshops. ESWC 2011. Lecture Notes in Computer Science (vol. 7117). Berlín: Springer.

Small, T. (2010). Canadian politics in 140 characters: Party politics in the Twitterverse. Canadian Parliamentary Review, 33(3), 39-45.

Straus, J. R., Glassman, M. E., Shogan, C. J. y Smelcer, S. N. (2013). Communicating in 140 characters or less: Congressional adoption of Twitter in the 111th Congress. PS: Political Science \& Politics, 46(1), 60-66. https://doi.org/10.1017/S1049096512001242

Tajel, H. y Turner, J. (1979). An integrative theory of intergroup conflict. En W. Austin y S. Worchel (eds.), The social psychology of intergroup relations (pp. 33-47). Monterey: Brooks/Cole.

Thompson, J. (1998). Los media y la modernidad: una teoría de los medios de comunicación. Barcelona: Paidós.

Túñez, M. y Sixto, J. (2011). Redes sociales, política y compromiso 2.0: la comunicación de los diputados españoles en Facebook. Revista Latina de Comunicación Social, 66, 1-25. https://doi.org/10.4185/ RLCS-66-2011-930-210-246

Velasco, J. D. (2017). El voto uribista en los municipios colombianos: patrones y significados (2002-2014). Análisis Político, 30(89), 3-37.

Wattenberg, M. P. (1982). From parties to candidates: Examining the role of the media. Public Opinion Quarterly, 46(2), 216-227.

Wodak, R. y Koller, V. (2008). Handbook of communication in the public sphere. Berlín: Walter de Gruyter. 